\title{
A Simple Model of "Creativity" and the Transformation of Economic Growth Mode
}

\author{
Chen Yi \\ Xiamen University, Xiamen City, Fujian Province, China \\ chyionly@qq.com
}

Keywords: creativity, the mode of economic growth, increasing returns to scale

\begin{abstract}
From Adam-Smith to Samuelson, the economic theory is adapted to the industrial economy. While the era of knowledge economy comes, the traditional economic theory has no longer met the requirements of the knowledge economy. As in known to us, in the traditional economics, the classical function of production and the model of economic growth usually analyze the impact of factors like capital or labor on economic growth, through the way of increasing the inputs of capital or labor. However, the contributions of creativity, technology and culture to the economic growth have enhanced, through the way of increasing the productivity, which has made the traditional growth theory occur a crisis. This paper tries to discuss how "creativity" transforms the mode of economic growth via a growth model that contains "creativity", on the basis of that "creativity" enables the function of production to exist an important feature of increasing returns to scale.
\end{abstract}

\section{Introduction}

The mode of economic growth is defined as the way and means of achieving the economic growth. The transformation of the mode of economic growth mainly refers to that the economic growth will be from extensive mode to intensive one, from epitaxial growth to organic growth. Generally the economic growth can be divided into two parts, one is caused by increasing the amount of factor inputs, the other is to increase the factor productivity. The extensive mode mainly depends on increasing inputs to achieve greater growth while the intensive growth is by way of increasing factor productivity to achieve greater growth. The traditional economics is majored in research on capital and labor's impact on economic growth, but in the era of knowledge economy, cultural creativity and technologic innovation are the tow cores of the knowledge economy. The features of creativity enables the improvement of factors' productivity, and thus achieves the transformation of the mode of economic growth.

\section{The "creativity": Produces effect of increasing returns to scale}

In most of today's discussion, "creativity" is associated with a variety of claims as culture, knowledge, information, innovation etc., and these references are often substituted for each other. And what is the "creativity"? "Creativity" is actually "have the ideas", and it depends on a person's capacity of dreaming, imagining, thinking, questioning and so on. Romer (1993) has stated that commodity is divided into two kinds, namely creativity (ideas) and objects. In a sense, "creative" are the commands on how to organize and produce utility. We know that, silica has always been existed as sands in a long time, but now it has become an indispensable element for making computer chips when a better way to organize the elements was found. Nowadays "creative" has become a new driving force for the sustainable development of economy. Usually creative process is to manifest culture by means of technical innovation.

As can be seen from the above description, "creativity" for economic development has a very important significance. So what is the mechanism with regard to the role of creativity in promoting economic growth? The relationship between "creativity" and the economic development can be described as follows:

Creativity (Ideas) $\rightarrow$ noncompetitive (Nonrivalry) $\rightarrow$ increasing returns to scale (IRS) $\rightarrow$ 
competitive equilibrium problem (fail) (Problems with CE). ${ }^{1}$

The first arrow in the illustration shows the characteristic of "creativity" - noncompetitive. In the public economics, a person's use of a noncompetitive product will not affect others' use of this product. Now we consider a function of production:

$$
Y=F(A, X) \text {. }
$$

Wherein A represents creative inputs, $\mathrm{X}$ represents other factors of production inputs such as capital, labor and so on. Suppose we want to produce twice the amount of Y, one means is to repeat inputs of $\mathrm{X}$, another means is to use "creative" stock which is noncompetitive (already existing creativity can be used in new factories or by new workers). We can use the model to express the views:

$$
\begin{aligned}
& \text { Supposing } \quad \lambda>1, \\
& F(A, \lambda X)=\lambda Y . \\
& F(\lambda A, \lambda X)>\lambda Y .
\end{aligned}
$$

For competitive factor $\mathrm{X}$, it shows the constant returns to scale, while for $\mathrm{X}$ and A overall, it shows increasing returns to scale. So if we increase $X$ and $A$ with the times, we can get more multiple outputs. It is thus clear that the factor of "creativity" produces the effect of increasing returns to scale.

The last arrow of illustration is "competitive equilibrium problem (fail)". The competitive equilibrium requires the marginal product for each factor. However, in the case of increasing returns to scale, obviously it is not true. Consider the production function (1), for X:

$$
F_{X} X=Y \text {. }
$$

As we know, the "competitive" factors of production which is paid for their marginal product, have just exhausted all the outputs. But once introduced "creativity" and paid for its marginal product, then:

$$
F_{X} X+F_{A} A>Y \text {. }
$$

It means the competitive equilibrium fails. The two main features of increasing returns to scale and competitive equilibrium failure have become the basis of the growth model with "creativity" below.

\section{The "creativity": Promotes transformation of economic growth mode}

In this section we will adopt a growth model $^{2}$ which contains "creativity" to further explore how "creativity" promotes transformation of the mode of economic growth. To make the model more intuitively represented, we assume there is only a unique competitive factor of production: labor, and it produces only a single consumption goods. The function of production is as follows:

$$
Y_{t}=A_{t}^{\sigma} L_{Y_{t}}, \quad \sigma>0
$$

Where $\mathrm{Y}$ is the total output, A is the creative input, $\boldsymbol{L}_{\boldsymbol{Y}}$ is the workers engaged in producing goods. Obviously, when A is constant, the function shows constant returns to scale, and when A is variable, it shows increasing returns to scale.

In addition, the "creativity" A can be considered as a kind of "product", and the function of production is as follows:

$$
\dot{A}_{t}=v\left(A_{t}\right) L_{A_{t}} A_{t}^{\phi}, \quad v>0
$$

$\dot{A}$ refers to the new creative amount increased in period t, $L_{A}$ means the researchers who produce new creativity. And the production constraints are:

$$
L_{Y}+L_{A}=L \text {. }
$$

\footnotetext{
${ }^{1}$ Refer to: Charles I. Jones, “Growth and Ideas”, Handbook of Economic Growth.

2 Mainly refer to: Charles I. Jones, "Growth and Ideas", Handbook of Economic Growth.
} 


$$
L_{t}=L_{0} e^{n t}, \quad n>0 .
$$

It is assumed that the proportion of the researchers in the total labor force is s, so the proportion of the labor force engaged in the production is (1-s). Based on the assumption we try to solve the problem of the growth about income per capita.

Firstly, from equation (7) can be obtained:

$$
y_{t}=\frac{Y_{t}}{L_{t}}=\frac{A_{t}^{\sigma} L_{Y_{t}}}{L_{t}}=(1-s) A_{t}^{\sigma} .
$$

From (10) it can be seen that the output is growing with the "creativity" stock per capita. As "creativity" is of noncompetitive feature, the output per capita depends on "creativity" total rather than the per capita amount. According to (10):

$$
\frac{\dot{y}_{t}}{y_{t}}=\sigma \frac{\dot{A}_{t}}{A_{t}} \text {. }
$$

The equation(11) shows that the growth rate of output per capita is proportional to the growth rate of the creativity stock. $\sigma$ measures the size of increasing returns. According to (7):

$$
\frac{\dot{A}_{t}}{A_{t}}=v \frac{L_{A_{t}}}{A_{t}^{1-\phi}} \text {. }
$$

Because $\phi<1$, it means that there will be a stably balanced path for economic growth (all variables are with the same growth rate). Then solving the model we have:

$$
g_{A}=\frac{n}{1-\phi} \text {. }
$$

Therefore, the growth rate of output per capita is:

$$
g_{y}=\sigma g_{A}=\frac{\sigma n}{1-\phi} \text {. }
$$

The growth rate of output per capita is proportional to the growth rate of population, and the parameters depend on the relative degree of increasing returns between the two sectors.

\section{Conclusion}

From the simple model, we found that when "creativity" as a factor is introduced into the function of production, a long-term economic growth can be realized and it depends on the relative degree of increasing returns to scale between production sector and R\&D sector. The two sectors' increasing returns to scale are both brought by the "creativity". In a word, the "creativity" that enables the function of production the feature of increasing returns to scale, will change the mode of economic growth, and finally achieve a long-term and stably economic growth.

\section{References}

[1] Amabile: Creativity in context, Boulder Westview Press(1996).

[2] The Creative Economy Report (2008).

[3] Charles I. Jones: Growth and Ideas, Handbook of Economic Growth.

[4] Robert E.Lucas,Jr: Ideas and Growth, National Bureau of Economic Research Working Paper,(2008).

[5] Wuwei Li: Creativity changes China, Xinhua Press(2009). 